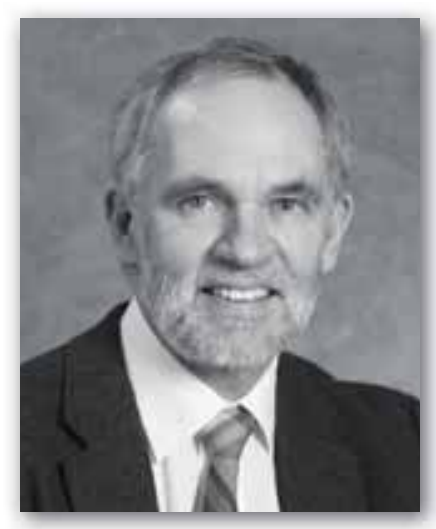

\author{
Editor, Anton Doubell \\ Professor and Head of the Division of Cardiology, Department of Medicine, \\ Faculty of Medicine and Health Sciences, Stellenbosch University and \\ Tygerberg Hospital, South Africa
}

\title{
The left atrial appendage - a design flaw or an essential structure?
}

The left atrial appendage (LAA) is considered to be a vestigial remnant of the primitive foetal atrium. It has also been identified as the source of the majority of left atrial thrombi in nonvalvular atrial fibrillation (AF). Not having a clearly defined and recognised function, and having the reputation of being the villain in thrombotic stroke in patients with non-valvular AF, has led to a number of rather aggressive interventions targeted at dealing with the scourge of AF.

The LAA has been burnt, slashed, lassoed and even had its throat stuffed, all without a thought for its wellbeing or consideration for the possibility that it may have an important function to play and that these interventions could impact on its function. So, should the LAA not take kindly to the way it is being treated and should it decide to fight back, it may very well lay charges of assault and attempted murder. The council for the defence would no doubt put forward 3 strong arguments justifying the alleged assault on the LAA.

The strength of the defence council's case would centre on the benefits of excluding the LAA from the circulation. Patients in AF have a 5 -fold increase in stroke. As many as $90 \%$ of these strokes are thought to result from emboli originating in the LAA. Although the stroke risk can be effectively reduced with anti-coagulant therapy, patient non-compliance often prevents this from being achieved and due to the bleeding risk associated with the use of anti-coagulants, there are patients in whom anti-coagulant use is not an option. An alternative approach is therefore to isolate the LAA, being the main source of emboli in patients with AF, for stroke prevention. (1) The PROTECT AF and the PREVAIL trials reporting on the use of the Watchman device in patients who were still eligible to receive warfarin indicate that it offers a non-inferior treatment strategy for stroke prevention in patients with non-valvular AF. It is a logical assumption that LAA exclusion would be the preferred strategy in patients who cannot tolerate warfarin, or a NOAC, but this is yet to be proven in clinical trials. Council for the defence would take comfort in the strength of the compelling evidence justifying the assaults on the LAA. The plaintive may well argue that the procedure runs the risk of life threatening complications with $2.3 \%$ of patients undergoing the Lariat procedure requiring urgent cardiac surgery. That is the risk for the individual but the risk is thought to be lower with the Watchman device. The council for the defence would remind us that the evidence for the cohort, as a whole, indicates that the procedural risks are outweighed by the benefits of excluding the LAA as a source of embolic stroke. 
The council for the defence would also argue that the LAA is the weakling of atrial society, not contributing to its mechanical function and that the atrial society would be better off without it. There is some evidence that atrial hypo-contractility, as part of an atrial cardiomyopathy, (2) characterised by increased fibrosis, may precede the development of AF, but it is not known if the atrial appendage impacts positively, negatively or not at all on atrial mechanical function. It would therefore be prudent not to dismiss a potential positive role for the LAA in the setting of an atrial cardiomyopathy until we fully understand its role.

The council for the defence would also have us believe that the LAA is just a bit of muscle and, as it has no other function, the remaining atrial muscle can easily compensate for its loss. After all, it is just a muscle and in patients with AF, a weak muscle at that. Given the dastardly deeds that it is known for, harbouring fugitive thrombi, giving these villains safe passage until they are allowed to flee and wreak havoc in the cerebral circulation, atrial society is surely better off without it. The council for the defence would no doubt be worried to learn that there is a growing recognition that the puny LAA may indeed pack a punch in the heavyweight division of cardiovascular homeostasis. Atrial specific granules, the storage vessels for atrial natriuretic peptide (ANP), are present in large numbers in the atrial appendage of patients with $A F^{(3)}$ As much as $30 \%$ of the ANP produced by the atria have the atrial appendages as its source. Does this store of ANP represent redundancy of this important cardiac endocrine system or are we overlooking an important homeostatic function of the LAA? The recent publication of the LAA HOMEOSTASIS trial has sparked renewed interest in the neuro-endocrine role of the LAA. ${ }^{(4)}$ The investigators monitored an impressive array of neuro-endocrine markers in patients undergoing LAA exclusion and compared the results in 2 groups. The first group had their LAA excluded by an epicardial technique using the Lariat device that achieves LAA exclusion through a percutaneous suture or lasso. In this group the LAA was ultimately destroyed (sent to the gallows) through a process of atrophy and replaced by fibrosis. The second group had their LAA excluded by plugging the ostium of the LAA (sent into exile) with a Watchman device. In this group the LAA was excluded from the left atrial chamber but its anatomical structure and endocrine components presumably remains intact. They reported profound differences in the neuro-endocrine sequelae of the two procedures involving the atrial natriuretic system (ANP and BNP levels), the renin-angiotensin system (renin and aldosterone levels) and the sympathetic nervous system (adrenalin and nor-adrenaline levels). This included, as would be predicted from our existing knowledge, an immediate decrease in ANP levels when using the Lariat device and an immediate increase in ANP levels when using the Watchman device for LAA exclusion. More unexpected was the finding of a persistent decrease in the levels of adrenaline, 
Editor, Anton Doubell noradrenaline, renin and aldosterone, accompanied by a sustained and significant decrease in blood pressure in patients in whom the Lariat device had been used. Have they identified a new approach to the management of resistant hypertension or have they alerted us to the dangers of opening, or rather closing, Pandora's box.

There is no doubt that the interventional approach to reduce stroke risk in patients with AF has added an important strategy to existing anti-coagulant based strategies for stroke prevention. The interventional community must be applauded for taking this bold step to increase our therapeutic options in these patients. However, a word of caution is required. When we use a LAA exclusion device we impact on cardiovascular homeostasis in ways that we do not yet fully understand. Will we learn that the impact on these homeostatic systems are trivial and of little clinical importance? Or will be learn that the long term effects of interfering with this apparently vestigial system comes at a cost? I wish that current evidence would allow me to say "I rest my case". However, current evidence, or the lack thereof forces me to say "Watch this space".

\section{REFERENCES}

I. Nishimura N, Sab S, Reeves RR, Hsu JC. Percutaneous left atrial appendage occlusion in atrial fibrillation patients with a contraindication to oral anticoagulation: A focused review. Europace 2017;0:1-8.

2. Guichard J-B, Nattel S. Atrial Cardiomyopathy. A useful notion in cardiac disease management of a passing fad? JACC 2017:6:756-765.

3. Doubell AF, Greef M, Rossouw D, Weich HF. Electron microscopic analysis of the specific granule content of human atria. An investigation of the role atrial pressure and atrial rhythm in the release of atrial natriuretic peptide. S Afr Med J 1990;78(4):207-2011.

4. Lakkireddy D, Turugam M, Afzal MR, et al. Left atrial appendage closure and systemic homeostasis. The LAA HOMEOSTASIS study. JACC 20 I 8;7I (2): I35- I 44. 